\title{
Evaluación de la Calidad Técnica Científico en el Servicio de Emergencia del Hospital Abel Gilbert Pontón Año 2018 - 2019 (1)(9)(
}

Evaluation of Scientific Technical Quality in the Emergency Service of the Abel Gilbert Pontón Hospital Year 2018 - 2019

Rosa Angélica Guerrero Lambida ${ }^{1}$

Recibido: 10-02-2019 / Revisado: 15-02-209 /Aceptado: 04-03-2019/ Publicado: 27-04-2019

\begin{abstract}
.
DOI: https://doi.org/10.33262/cienciadigital.v3i2.1.434

The continuous improvement of the quality of care within the national health systems is one of the main objectives for the MSP and private institutions. One of the components to be evaluated is the technical-scientific quality to achieve the efficiency, effectiveness and effectiveness of the evaluated service, for which several identification and problem assessment tools have been developed, one of them being health indicators.

This study was a retrospective cross-sectional type of analysis, in which the technicalscientific quality of the emergency area is evaluated in an objective way by means of indicatorsThe universe of 1550 patients attended in 6 months, a probabilistic sample of 300 clinical cases taken at random was taken from the 10 most frequently attended pathologies in the emergency area of the Abel Gilbert Pontón specialties hospital with its respective protocol; the information obtained in digital formats 008 and 005 during the period of July 2018 to January 2019 was considered.

The sample was composed of 300 clinical histories, the values obtained will be the numerators and the denominators of the indicators developed for the scientific technical evaluation of the emergency area with an initial standard value greater than $70 \%$ as optimal according to the Spanish guidelines of Andalusia and WHO which were taken as reference on quality of care.
\end{abstract}

\footnotetext{
${ }^{1}$ Médico General, Hospital Abel Gilbert Pontón Ecuador, email rosa amadaguelo@ yahoo.com
} 
As a result, it was found that the evaluation made of the technical-scientific quality of the emergency service identified the delay in decision-making in relation to the final behavior with patients as the main problem, leading to a longer stay at 6 hours, a value that it is not in relation to an optimal quality of care and that it would be related to the lack of socialization of the service protocols and updating them that were other problems identified, the next step is the preparation of action plans as part of the cycle of continuous improvement in the quality of care.

Keywords: Medical Audit, Quality Of Care, Health Services, Health Indicators, Continuous Improvement Cycle

\section{Resumen.}

La mejora continua de la calidad asistencial dentro de los sistemas nacionales de salud es uno de los objetivos principales para el MSP e instituciones privadas. Uno de los componentes a evaluar es la calidad técnico científica para lograr la eficiencia, eficacia y efectividad del servicio evaluado para lo cual se han elaborado varias herramientas de identificación y evaluación de problemas, siendo uno de ellos los indicadores de salud.

Este estudio fue de tipo transversal retrospectivo de carácter analítico, en el que se evalúa la calidad técnico científico del área de emergencia de una forma objetiva por medio de indicadores

El universo de 1550 pacientes atendidos en 6 meses, se tomó una muestra probabilística de 300 casos clínicos tomados al azar, de las 10 patologías más frecuentemente atendidos en el área de emergencia del hospital de especialidades Abel Gilbert Pontón con su respectivo protocolo; se consideró la información obtenido en los formatos digitálicos 008 y 005 durante el periodo de julio del 2018 hasta enero del 2019.

La muestra fue compuesta por 300 historias clínicas, los valores obtenidos serán los numeradores y los denominadores de los indicadores elaborados para la evaluación técnica científica del área de emergencia con un valor estándar inicial mayor del 70\% como óptimo según las guías españolas de Andalucía y OMS que se tomaron como referencia sobre calidad asistencial.

Como resultado se obtuvo que la evaluación realizada de la calidad técnico científico del servicio de emergencia identificó como principal problema la demora en la toma de decisiones en relación a la conducta definitiva con los pacientes, conduciendo a una estancia mayor a las 6 horas, valor que no está en relación a una calidad asistencial 
optima y que se relacionaría con la falta de socialización de los protocolos del servicio y actualización de los mismos que fueron otros de los problemas identificados, el siguiente paso es la elaboración de planes de acción como parte del ciclo de mejora continua en la calidad asistencial.

Palabras clave: auditoria médica, calidad asistencial, servicios de salud, indicadores de salud, ciclo de mejora continua

\section{Introducción.}

El brindar una calidad asistencial de excelencia consiste en "hacer bien lo que se debe de hacer" en cada proceso asistencial, en el momento oportuno, con los recursos disponibles y con el menor coste posible para causar la satisfacción de quien la recibe. El alcanzar este objetivo requiere la participación conjunta y multidisciplinaria de profesionales entre los que participan directamente con el usuario (operativos), los que planifican (gestores) y el mismo usuario (población) lo que significa conseguir la calidad técnica científica y la calidad relacional. (Pérez \& Arnesto, 2015)

La calidad científico-técnica relaciona la efectividad de los resultados obtenidos con eficacia, empleando el esquema más eficiente de las guías de práctica clínica de una forma accesible, equitativa y segura en los procesos atendidos. (Pérez \& Arnesto, 2015)

La calidad relacional se enfoca principalmente en la capacidad de comunicarse con quienes reciben los servicios, basándose en los principios y valores de ética asistencial además de las preferencias de las personas que reciben la asistencia. (Pérez \& Arnesto, 2015)

Una vez entendida la definición de calidad asistencial y sus componentes, el objetivo de este trabajo es aplicar de una forma práctica una de las técnicas de evaluación disponibles para identificar los posibles problemas en uno de los componentes de la calidad asistencial, como es la calidad técnica - científico y establecer el nivel de calidad estándar como estrategia para la mejora continua.

\section{Metodologia.}

La metodología utilizada en el estudio es de tipo transversal retrospectivo de carácter analítico, en el que se evalúa la calidad técnico científico del área de emergencia de una forma objetiva por medio de indicadores 


\section{Universo y muestra:}

Para el desarrollo de esta evaluación, se utilizó una muestra probabilística de 300 casos clínicos de las 10 patologías más frecuentemente atendidos en el área de emergencia del hospital Abel Gilbert Pontón; se consideró la información obtenido en los formatos digitálicos 008 y 005 durante el periodo 2018 - 2019

\section{Criterios de inclusión}

- Todas las edades y géneros

- Las historias clínicas del área de emergencia

- Las 10 patologías más frecuentes evaluadas en el área de emergencia por médicos de emergencia

\section{Criterios de exclusión:}

- Las historias clínicas de consulta externa

- Las evaluaciones por médicos o especialidades que no corresponden al aérea de emergencia

- Otras patologías que no estén dentro del Rankin de las 10 patologías más frecuentes

\section{Tipo de estudio:}

Es un estudio de tipo transversal retrospectivo de carácter evaluativo, en el que se evalúa la calidad técnico científico del área de emergencia de una forma objetiva por medio de indicadores.

Variables:

Estructura:

- Existencia de protocolos médicos en físico en el área de emergencia

- Disponibilidad de equipos médicos y reactivos de laboratorio idóneos para la emergencia

- Disponibilidad de insumos médicos y medicamentos para los procedimientos del área de emergencia

\section{Proceso:}

- Cumplimiento de los protocolos médicos establecidos para el área de emergencia 
- Conocimiento de los protocolos por los profesionales.

- Pertinencia de las pruebas complementarias solicitadas por la unidad

- Grado de correlación entre el diagnostico de ingreso y el diagnostico de alta

MATRIZ DE OPERACIONALIZACION Y CRUCES DE INDICADORES

\begin{tabular}{|c|c|c|c|c|}
\hline $\begin{array}{l}\text { DESCRIPCION } \\
\text { DEL PROCESO }\end{array}$ & JUSTIFICACION & INDICADOR & FORMULA & ESTÁNDAR \\
\hline $\begin{array}{l}\text { EXISTENCIA } \\
\text { FISICA DE } \\
\text { PROTOCOLOS } \\
\text { MEDICOS EN EL } \\
\text { AREA DE } \\
\text { EMERGENCIA }\end{array}$ & $\begin{array}{l}\text { La existencia de } \\
\text { protocolos en el área de } \\
\text { emergencia elaborados } \\
\text { en base a las guías } \\
\text { medicas estandarizadas } \\
\text { para unificar criterios }\end{array}$ & $\begin{array}{l}\text { Existencia de } \\
\text { protocolos } \\
\text { médicos en físico } \\
\text { en el área de } \\
\text { emergencia }\end{array}$ & $\begin{array}{l}\text { SI } \\
\text { NO }\end{array}$ & Estándar 95\% \\
\hline $\begin{array}{l}\text { NÚMERO DE } \\
\text { PROFESIONALES } \\
\text { SOCIALIZADOS } \\
\text { CON LOS } \\
\text { PROTOCOLOS DE } \\
\text { LA INSTITUCIÓN }\end{array}$ & $\begin{array}{l}\text { Es deber de la } \\
\text { institución socializar } \\
\text { los protocolos del área } \\
\text { para hablar el mismo } \\
\text { idioma }\end{array}$ & $\begin{array}{l}\text { Conocimiento de } \\
\text { los protocolos por } \\
\text { los profesionales. }\end{array}$ & $\begin{array}{l}\text { Numerador } \\
\mathrm{N}^{\circ} \text { profesionales } \\
\text { que conocen los } \\
\text { protocolos } \\
\text { Denominador } \\
\text { Número total de } \\
\text { profesionales }\end{array}$ & $\begin{array}{l}\text { Estándar del } \\
95 \%\end{array}$ \\
\hline $\begin{array}{l}\text { CUMPLIMIENTO } \\
\text { DE } \\
\text { PROTOCOLOS } \\
\text { MÉDICOS } \\
\text { ESTABLECIDOS } \\
\text { PARA EL ÁREA DE } \\
\text { EMERGENCIA DEL } \\
\text { HAGP } \\
\text { ACUERDO A LOS } \\
\text { ESTANDARES DE } \\
\text { CALIDAD }\end{array}$ & $\begin{array}{l}\text { El desarrollo de } \\
\text { protocolos basados en } \\
\text { la evidencia clínica } \\
\text { disponible, y evaluado } \\
\text { por los profesionales, } \\
\text { es una herramienta } \\
\text { fundamental para el } \\
\text { mantenimiento y } \\
\text { mejora de la calidad }\end{array}$ & $\begin{array}{l}\text { Cumplimiento de } \\
\text { protocolos, } \\
\text { procedimientos, } \\
\text { guías de práctica } \\
\text { clínica y planes de } \\
\text { cuidados }\end{array}$ & $\begin{array}{l}\text { Numerador: } \mathrm{N}^{\circ} \\
\text { de historias } \\
\text { clínicas que } \\
\text { cumplen con los } \\
\text { indicadores de } \\
\text { calidad técnico } \\
\text { científica } \\
\text { Denominador: } \\
\mathrm{N}^{\circ} \text { total de } \\
\text { historias clínicas } \\
\text { del servicio de } \\
\text { emergencias }\end{array}$ & $\begin{array}{l}\text { ESTÁNDAR } \\
80 \%\end{array}$ \\
\hline $\begin{array}{l}\text { PERTINENCIA DE } \\
\text { LAS PRUEBAS } \\
\text { COMPLEMENTARI } \\
\text { AS SOLICITADAS } \\
\text { POR LA UNIDAD }\end{array}$ & $\begin{array}{l}\text { La solicitud y análisis } \\
\text { de exámenes } \\
\text { complementarios en el } \\
\text { momento oportuno y } \\
\text { los indicados expresa } \\
\text { sobre la eficacia de la } \\
\text { unidad y sus costes }\end{array}$ & $\begin{array}{l}\text { PERTINENCIA } \\
\text { DE LAS } \\
\text { PRUEBAS } \\
\text { COMPLEMENT } \\
\text { ARIAS } \\
\text { (LABORATORI } \\
\text { O E IMAGEN ) E } \\
\text { INTERCONSUL } \\
\text { TAS } \\
\text { SOLICITADAS } \\
\text { ACORDE AL } \\
\text { DIAGNOSTICO }\end{array}$ & $\begin{array}{l}\text { SI } \\
\text { NO }\end{array}$ & $\begin{array}{l}\text { ESTANDAR } \\
95 \%\end{array}$ \\
\hline
\end{tabular}


ISBN 2602-8085

Vol. 3, $\mathrm{N}^{\circ} 2.1$, p. 93-107, abril - junio, 2019

\begin{tabular}{|c|c|c|c|c|}
\hline $\begin{array}{l}\text { GRADO DE } \\
\text { CORRELACION } \\
\text { ENTRE EL } \\
\text { DIAGNOSTICO DE } \\
\text { INGRESO Y EL } \\
\text { DIAGNOSTICO DE } \\
\text { ALTA }\end{array}$ & $\begin{array}{l}\text { Es necesario que el } \\
\text { diagnóstico del alta sea } \\
\text { lo más preciso posible } \\
\text { para evitar errores con } \\
\text { desenlace fatales }\end{array}$ & $\begin{array}{l}\text { Concordancia } \\
\text { diagnostica }\end{array}$ & $\begin{array}{l}\text { SI } \\
\text { NO }\end{array}$ & $\begin{array}{l}\text { ESTÁNDAR } \\
80 \%\end{array}$ \\
\hline $\begin{array}{l}\text { TIEMPO MEDIO } \\
\text { DE PRIMERA } \\
\text { ATENCIÓN } \\
\text { MEDICA EN } \\
\text { URGENCIAS }\end{array}$ & $\begin{array}{l}\text { Uno de los puntos } \\
\text { clave para la } \\
\text { satisfacción de los } \\
\text { usuarios es la rapidez } \\
\text { con la que un médico } \\
\text { atiende y evalúa al } \\
\text { paciente Este indicador } \\
\text { permite conocer la } \\
\text { eficacia y eficiencia del } \\
\text { médico y de la } \\
\text { organización del } \\
\text { proceso para evitar que } \\
\text { las patologías graves } \\
\text { no se vean afectadas } \\
\text { negativamente por } \\
\text { demoras en la } \\
\text { asistencia inicial. }\end{array}$ & $\begin{array}{l}\text { Tiempo medio de } \\
\text { primera atención } \\
\text { médica en } \\
\text { urgencias }\end{array}$ & $\begin{array}{l}\text { Numerador: } \\
\text { Suma del } \\
\text { tiempo de } 1^{\text {a }} \\
\text { asistencia de } \\
\text { cada uno de los } \\
\text { pacientes } \\
\text { atendidos en un } \\
\text { período de } \\
\text { tiempo dado } \\
\text { Denominador: } \\
\text { Número total de } \\
\text { pacientes } \\
\text { atendidos en } \\
\text { dicho período } \\
\text { de tiempo. } \\
\text { (Se tomará en } \\
\text { cuenta los } \\
\text { tiempos de triaje } \\
\text { Manchester) }\end{array}$ & $\begin{array}{l}\text { Estándar } \\
90 \%\end{array}$ \\
\hline $\begin{array}{l}\text { TIEMPO DE } \\
\text { PERMANENCIA DE } \\
\text { LO PACIENTES EN } \\
\text { EL SERVICIO DE } \\
\text { EMERGENCIAS }\end{array}$ & $\begin{array}{l}\text { La identificación y } \\
\text { solución del problema } \\
\text { por el cual va el } \\
\text { paciente a la } \\
\text { emergencia debe ser } \\
\text { dentro de un tiempo } \\
\text { óptimo. Un tiempo de } \\
\text { estancia prolongado en } \\
\text { el área de emergencia } \\
\text { indica que existen } \\
\text { déficits de } \\
\text { coordinación, circuitos } \\
\text { inadecuados, servicios } \\
\text { de soporte } \\
\text { insuficientes, etc. En } \\
\text { este caso, cada hospital } \\
\text { debería establecer sus } \\
\text { indicadores internos } \\
\text { para averiguar las } \\
\text { causas del incremento } \\
\text { de la permanencia. }\end{array}$ & $\begin{array}{l}\text { Tiempo de } \\
\text { conducta } \\
\text { definitiva con el } \\
\text { paciente en } \\
\text { servicio de } \\
\text { emergencias }\end{array}$ & $\begin{array}{l}\text { Numerador: } \mathrm{N}^{\circ} \\
\text { de pacientes } \\
\text { cuya conducta } \\
\text { final se definió } \\
\text { en }<6 \text { horas } \\
\text { Denominador: } \\
\mathrm{N}^{\circ} \text { total de } \\
\text { pacientes } \\
\text { atendidos en } \\
\text { emergencia } \\
\text { dicho período. }\end{array}$ & Estándar 90\% \\
\hline $\begin{array}{l}\text { TASA } \\
\text { RETORNO DE LOS }\end{array}$ & $\begin{array}{l}\text { Se considera normal } \\
\text { hasta cierto punto el } \\
\text { reingreso de pacientes } \\
\text { debido a su mala } \\
\text { evolución, pero es }\end{array}$ & & & \\
\hline
\end{tabular}


ISBN 2602-8085

Vol. 3, N².1, p. 93-107, abril - junio, 2019

\begin{tabular}{|c|c|c|c|c|}
\hline $\begin{array}{l}\text { PACIENTES AL } \\
\text { SERVICIO DE } \\
\text { EMERGENCIAS } \\
\text { DENTRO DE LAS } \\
\text { PRIMERAS 72H }\end{array}$ & $\begin{array}{l}\text { menos cierto que } \\
\text { cuando la calidad } \\
\text { técnica de la misma no } \\
\text { es la óptima, es más } \\
\text { probable que algunos } \\
\text { pacientes no reciban el } \\
\text { diagnóstico o } \\
\text { el tratamiento } \\
\text { adecuado, o no hayan } \\
\text { sido detectados todos } \\
\text { los } \\
\text { problemas de salud, y } \\
\text { precisen por ello de } \\
\text { una nueva atención. } \\
\text { Por este motivo parece } \\
\text { pertinente medir y } \\
\text { monitorizar en el } \\
\text { tiempo este indicador } \\
\text { como reflejo indirecto } \\
\text { de la efectividad } \\
\text { real y definitiva del } \\
\text { servicio de emergencia } \\
\text { y da la calidad de su } \\
\text { asistencia. }\end{array}$ & Tasa de reingreso & $\begin{array}{l}\text { Numerador: } \mathrm{N}^{\circ} \\
\text { de pacientes que } \\
\text { reingresan a las } \\
72 \mathrm{~h} \\
\text { Denominador: } \\
\mathrm{N}^{\mathrm{o}} \text { total de } \\
\text { pacientes } \\
\text { atendidos en } \\
\text { emergencia }\end{array}$ & Estándar 15\% \\
\hline
\end{tabular}

\section{Resultados.}

Para el desarrollo de esta evaluación se tomó una muestra de 300 historias clínicas al azar del área de emergencias del hospital de especialidades Abel Gilbert Pontón, con el objetivo de evaluar la calidad técnica científica de los profesionales médicos del área de forma cuantitativa la cual cuenta con un total de 57 médicos de los cuales 18 son especialistas, los valores obtenidos serán los numeradores y denominadores de los indicadores elaborados para dicha evaluación, con un valor estándar $>90 \%$ considerado como óptimo según las guías españolas de Andalucía y OMS que se tomaron como referencia sobre calidad técnica científica en calidad asistencial, obteniéndose los siguientes resultados:

\begin{tabular}{|l|l|}
\hline Nombre & $\begin{array}{l}\text { EXISTENCIA FISICA DE PROTOCOLOS MEDICOS EN } \\
\text { EL AREA DE EMERGENCIA }\end{array}$ \\
\hline Dimensión & Adecuación \\
\hline Justificación & $\begin{array}{l}\text { La existencia de protocolos en el área de emergencia } \\
\text { elaborados en base a las guías medicas estandarizadas para } \\
\text { unificar criterios }\end{array}$ \\
\hline
\end{tabular}


Vol. 3, N².1, p. 93-107, abril - junio, 2019

\begin{tabular}{|l|l|}
\hline Formula & SI o NO \\
\hline $\begin{array}{l}\text { Desarrollo de la formula y } \\
\text { resultados }\end{array}$ & $\begin{array}{l}\text { Si hay protocolos, pero no están actualizados } \\
\text { Puntaje: } 50 \%\end{array}$ \\
\hline Tipo & Proceso \\
\hline Fuente de datos & Información obtenida del jefe de emergencias \\
\hline Estándar & $>95 \%$ \\
\hline Comentarios & $\begin{array}{l}\text { Se dispone de protocolos, pero no están actualizados desde } \\
\text { hace 4 y 3 años }\end{array}$ \\
\hline
\end{tabular}

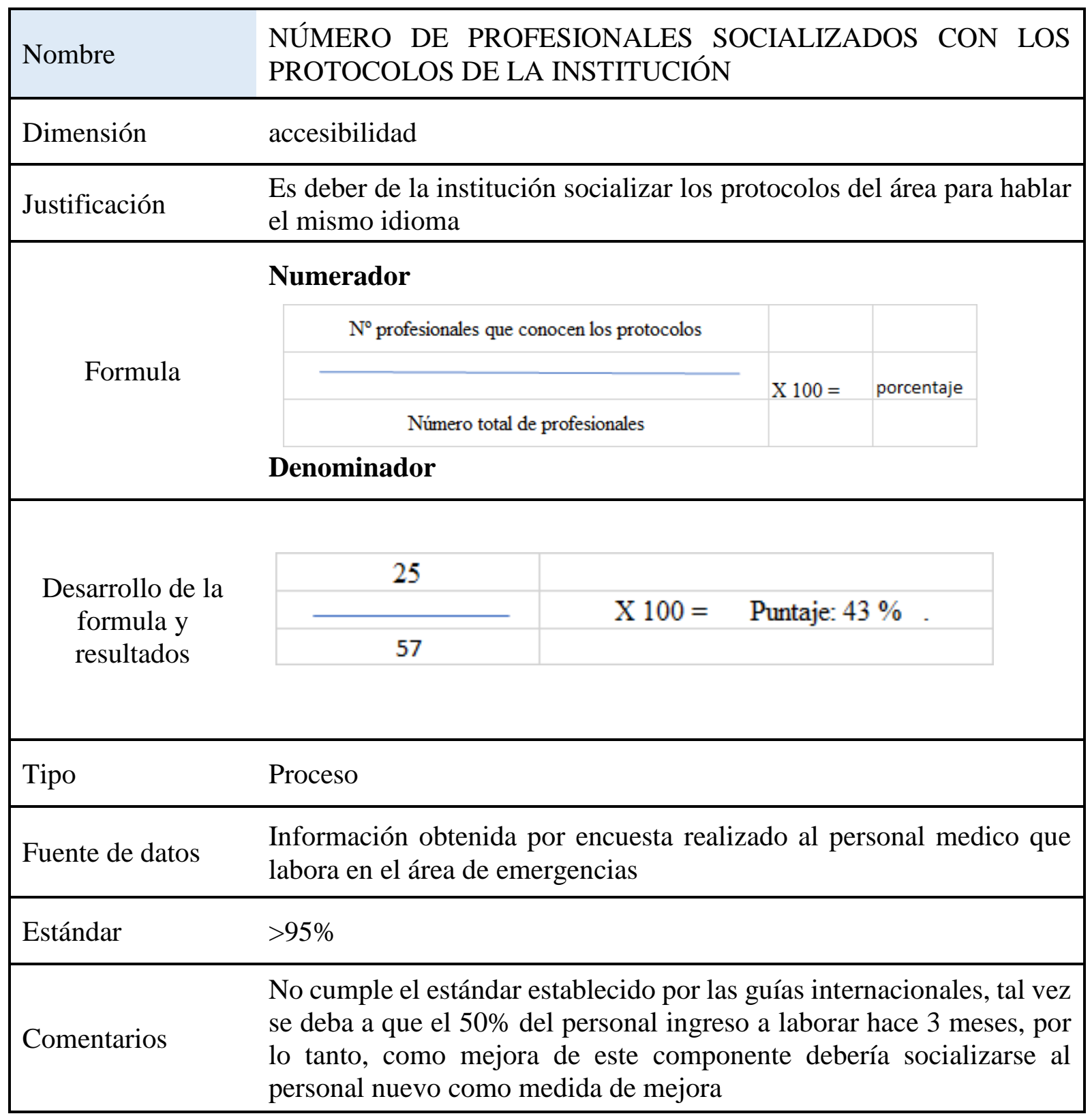


ISBN 2602-8085

Vol. 3, N².1, p. 93-107, abril - junio, 2019

\begin{tabular}{|c|c|}
\hline Nombre & $\begin{array}{l}\text { PERTINENCIA DE LAS PRUEBAS COMPLEMENTARIAS } \\
\text { (LABORATORIO E IMAGEN) E INTERCONSULTAS } \\
\text { SOLICITADAS ACORDE AL DIAGNOSTICO }\end{array}$ \\
\hline Dimensión & Eficacia \\
\hline Justificación & $\begin{array}{l}\text { La solicitud y análisis de exámenes complementarios en el } \\
\text { momento oportuno y los indicados expresa sobre la eficacia de la } \\
\text { unidad y sus costes }\end{array}$ \\
\hline \multirow[t]{2}{*}{ Formula } & $\begin{array}{l}\text { Numerador } \\
\mathrm{N}^{\circ} \text { de historias en la que hubo pertinencia en exámenes } \\
\text { complementarios }\end{array}$ \\
\hline & $\begin{array}{l}\text { Denominador } \\
\text { N } 100=\text { porcentaje } \\
\mathrm{N}^{\circ} \text { total de historias clínicas del servicio de emergencias }\end{array}$ \\
\hline $\begin{array}{l}\text { Desarrollo de la formula } \\
\text { y resultados }\end{array}$ & $\frac{220}{300} \times 100=$ Puntaje: $73 \%$ \\
\hline Tipo & Proceso \\
\hline Fuente de datos & $\begin{array}{l}\text { Se obtuvo de la correlación entre el diagnostico presuntivo de } \\
\text { ingreso en la historia clínica, la valoración clínica del galeno que } \\
\text { justifique los exámenes solicitados. }\end{array}$ \\
\hline Estándar & $>95 \%$ \\
\hline Comentarios & $\begin{array}{l}\text { De las } 300 \text { historias clínicas tomadas al azar solo } 220 \text { presentaban } \\
\text { cierto grado de correlación entre el diagnóstico, los hallazgos } \\
\text { clínicos y los exámenes solicitados alcanzando un porcentaje de } \\
73 \% \text { por lo tanto no alcanzo el estándar de calidad. }\end{array}$ \\
\hline
\end{tabular}

\begin{tabular}{|c|c|}
\hline Nombre & $\begin{array}{l}\text { GRADO DE CORRELACION ENTRE EL DIAGNOSTICO } \\
\text { DE INGRESO Y EL DIAGNOSTICO DE ALTA }\end{array}$ \\
\hline Dimensión & Continuidad, eficacia y eficiencia \\
\hline Justificación & $\begin{array}{l}\text { Es necesario que el diagnóstico del alta sea lo más preciso posible } \\
\text { para evitar errores con desenlace fatales }\end{array}$ \\
\hline Formula & $\begin{array}{l}\text { Numerador } \\
\mathrm{N}^{\circ} \text { de historias con dg de ingreso e ingreso relacionados } \\
\text { Denominador } \\
\mathrm{N}^{\circ} \text { total de historias clínicas del servicio de emergencias }\end{array}$ \\
\hline $\begin{array}{l}\text { Desarrollo de la formula } \\
\text { y resultados }\end{array}$ & $\frac{250}{300} \times 100=\mathbf{8 3 \%}$ \\
\hline
\end{tabular}


ISBN 2602-8085

Vol. 3, N².1, p. 93-107, abril - junio, 2019

\begin{tabular}{|l|l|}
\hline Tipo & Resultado \\
\hline Fuente de datos & $\begin{array}{l}\text { Se obtuvo de la correlación entre el diagnostico presuntivo de } \\
\text { ingreso y el de egreso. }\end{array}$ \\
\hline Estándar & $>80 \%$ \\
\hline Comentarios & $\begin{array}{l}\text { La relación que guarda entre el diagnostico de ingreso y el alta es } \\
\text { relevante ya que filia el tratamiento iniciado y definitivo al igual } \\
\text { que la conducta definitiva a seguir con lo que se logra valorar } \\
\text { además la eficacia y eficiencia del personal de salud, en esta } \\
\text { valoración se ha alcanzado el valor estándar. }\end{array}$ \\
\hline
\end{tabular}

\begin{tabular}{|c|c|}
\hline Nombre & $\begin{array}{l}\text { TIEMPO MEDIO DE PRIMERA ATENCIÓN MEDICA EN } \\
\text { URGENCIAS }\end{array}$ \\
\hline Dimensión & Eficiencia y eficacia \\
\hline Justificación & $\begin{array}{l}\text { Uno de los puntos clave para la satisfacción de los usuarios es la } \\
\text { rapidez con la que un médico atiende y evalúa al paciente Este } \\
\text { indicador permite conocer la eficacia y eficiencia del médico y } \\
\text { de la organización del proceso para evitar que las patologías } \\
\text { graves no se vean afectadas negativamente por demoras en la } \\
\text { asistencia inicial. }\end{array}$ \\
\hline Formula & $\begin{array}{l}\text { Numerador } \\
\text { Número de pacientes atendidos dentro del tiempo acorde } \\
\text { al triage de Mánchester } \\
100=\text { porcentaje } \\
\text { Denominador } \\
\text { Número total de pacientes atendidos en la emergencia. }\end{array}$ \\
\hline $\begin{array}{l}\text { Desarrollo de la formula } \\
\text { y resultados }\end{array}$ & $\frac{215}{300} \times 100=\quad$ Puntaje: $71 \%$ \\
\hline Tipo & Proceso \\
\hline Fuente de datos & $\begin{array}{l}\text { Se valoro de acuerdo a los tiempos establecidos en el triage de } \\
\text { Manchester según los datos obtenidos de la hoja de triage }\end{array}$ \\
\hline Estándar & $>90 \%$ \\
\hline Comentarios & $\begin{array}{l}\text { La satisfacción de los usuarios es uno de los objetivos de la } \\
\text { atención de calidad en salud, haciendo atribución a esto y como } \\
\text { una forma de priorizar la atención de los pacientes críticos y } \\
\text { disminuir la mortalidad en las áreas de emergencias se elaboró } \\
\text { este sistema de atención en base a tiempos de espera para la }\end{array}$ \\
\hline
\end{tabular}


ISBN 2602-8085

Vol. 3, N².1, p. 93-107, abril - junio, 2019

atención de los pacientes de acuerdo a su riesgo clínico permitiendo un manejo de flujo de pacientes de una manera eficaz y eficiente cuando hay una mayor demanda del servicio. De los 300 pacientes atendidos 215 recibieron la primera atención medica dentro del tiempo establecido según su complejidad con un porcentaje del $71 \%$ estando fuera de los estándares de calidad de atención el cual debe ser mayor del $90 \%$ como optima, pero por ser una valoración inicial se acepta $>70 \%$ pero que debería ser sometida a evaluaciones continua para mejora

\begin{tabular}{|c|c|}
\hline Nombre & $\begin{array}{l}\text { TIEMPO DE PERMANENCIA DE LO PACIENTES EN EL } \\
\text { SERVICIO DE EMERGENCIAS }\end{array}$ \\
\hline Dimensión & Efectividad \\
\hline Justificación & $\begin{array}{l}\text { La identificación y solución del problema por el cual va el } \\
\text { paciente a la emergencia debe ser solucionado dentro de un tiempo } \\
\text { óptimo. Un tiempo de estancia prolongado en el área de } \\
\text { emergencia indica que existen déficits de coordinación, circuitos } \\
\text { inadecuados, servicios de soporte insuficientes, etc. En este caso, } \\
\text { cada hospital debería establecer sus indicadores internos para } \\
\text { averiguar las causas del incremento de la permanencia. }\end{array}$ \\
\hline Formula & $\begin{array}{l}\text { Numerador } \\
\text { Número de pacientes cuya conducta final se definió } \\
\text { en }<6 \text { horas }\end{array}$ \\
\hline $\begin{array}{l}\text { Desarrollo de la formula } \\
\text { y resultados }\end{array}$ & $\frac{120}{80} \times 100=$ Puntaje: $40 \%$ \\
\hline Tipo & Resultados \\
\hline Fuente de datos & Historias Clínicas \\
\hline Estándar & $>90 \%$ \\
\hline Comentarios & $\begin{array}{l}\text { El tiempo que demora un galeno en tomar una decisión en cuanto } \\
\text { a la conducta definitiva de su paciente expone mucho en cuanto a } \\
\text { su experiencia y nivel de conocimiento por eso este es uno de los } \\
\text { indicadores mas utilizados para la evaluación de la calidad técnico } \\
\text { científico estableciéndose con un estándar del } 90 \% \text { como mínimo, } \\
\text { por lo que tampoco se cumple este parámetro en esta evaluación }\end{array}$ \\
\hline
\end{tabular}


Vol. 3, N².1, p. 93-107, abril - junio, 2019

\begin{tabular}{|c|c|}
\hline Nombre & $\begin{array}{l}\text { TASA DE RETORNO DE LOS PACIENTES AL } \\
\text { SERVICIO DE EMERGENCIAS DENTRO DE LAS } \\
\text { PRIMERAS 72H }\end{array}$ \\
\hline Dimensión & Efectividad \\
\hline Justificación & $\begin{array}{l}\text { Se considera normal hasta cierto punto el reingreso de pacientes } \\
\text { debido a su mala evolución, pero es menos cierto que cuando la } \\
\text { calidad técnica de la misma no es la óptima, es más probable que } \\
\text { algunos pacientes no reciban el diagnóstico o el tratamiento } \\
\text { adecuado, o no hayan sido detectados todos los } \\
\text { problemas de salud, y precisen por ello de una nueva atención. } \\
\text { Por este motivo parece pertinente medir y monitorizar en el } \\
\text { tiempo este indicador como reflejo indirecto de la efectividad } \\
\text { real y definitiva del servicio de emergencia y da la calidad de su } \\
\text { asistencia. }\end{array}$ \\
\hline Formula & $\begin{array}{l}\text { Numerador } \\
\text { Número de pacientes que regresaron antes de las } 72 \mathrm{~h} \\
\text { con la misma sintomatología } \\
\text { Denominador } \\
\text { Número total de pacientes atendidos en la emergencia. }\end{array}$ \\
\hline $\begin{array}{l}\text { Desarrollo de la formula } \\
\text { y resultados }\end{array}$ & $\frac{120}{80} \times 100=$ Puntaje: $0 \%$ \\
\hline Tipo & Resultados \\
\hline Fuente de datos & Historias Clínicas \\
\hline Estándar & $<15 \%$ \\
\hline Comentarios & $\begin{array}{l}\text { La tasa de reingreso es otro de los parámetros que se evalúa como } \\
\text { parte de la calidad técnico científico en el área de emergencia, se } \\
\text { ha dispuesto que sea dentro de las primeros } 72 \mathrm{~h} \text { de haber sido dado } \\
\text { el alta el paciente y que acuda con las mismas molestias con un } \\
\text { estándar menor del } 15 \% \text { como óptimo. En esta ocasión se alcanzó } \\
0 \text { casos de reingreso dentro de las primeros } 72 \mathrm{~h} \text {, la mayoría } \\
\text { regresaba a los } 15 \text { días, pero por otras molestias. }\end{array}$ \\
\hline
\end{tabular}

\section{Conclusiones.}

- Una vez realizada la evaluación de la calidad técnico científico del servicio de emergencia del hospital Abel Gilbert Pontón con los indicadores de salud elaborados en base a estándares establecidos a nivel internacional y posterior análisis de los datos, se identificó como principal problema la demora en la toma de decisiones en relación a la conducta definitiva con los pacientes, conduciendo a una estancia mayor 
a las 6 horas, valor que no está en relación a una calidad asistencial optima y que se relacionaría con la falta de socialización de los protocolos del servicio y actualización de los mismos que fueron otros de los problemas identificados, lo que predispondría a un uso inadecuado de recursos y áreas físicas del área, por lo que debería priorizarse estos problemas para posterior elaboración de un plan de acción de mejora continua y posterior ejecución del mismo.

- La realización de una correcta auditoría médica de una forma sistemática, realizado por profesionales con experiencia y conocimientos amplios en el campo, en este caso médicos especialistas quienes comparan la calidad actual con la calidad ideal de acuerdo a normas y criterios preestablecidos, puede mejorar de forma significativa la calidad del servicio de salud, permitiendo gestionar los riesgos, reclamos y conflictos.

- Esta herramienta puede identificar las áreas críticas y proponer soluciones que favorezcan tanto al cuerpo médico como a los pacientes y permitir mejorar la calidad de la atención y la práctica médica, reduciendo al mínimo la incidencia de errores, siendo una excelente herramienta en el plano educativo permitiendo la formación de profesionales idóneos para el área y perfeccionamiento de los mismos a futuro de acuerdo a la necesidad institucional, definiendo a la auditoria medica como una instancia formadora e educativa más que punitiva y sancionadora.

\section{Referencias bibliográficas.}

Arrea, C., Rodriguez, J., Cabezas, E., Lorie, L., \& Brull, J. (2000). www.binasss.sa.cr/revistas/hcr/n101976/art4.pdf. Obtenido de el hospital orientado a servicios de emergencia.

Correia Loureiro, S. M. (2010). CALIDAD Y SATISFACCIÓN EN EL SERVICIO DE URGENCIAS en PORTUGAL. Investigaciones Europeas de Dirección y Economía de la Empresa, Vol. 16, No 2, 2010, pp. 27-41, ISSN: 1135-2523.

Dr. Ramon REYES, M. (2016). http://emssolutionsint.blogspot.com/2016/07/4-edicion-delmanual-de-protocolos-y.html. Obtenido de http://emssolutionsint.blogspot.com/2016/07/4-edicion-del-manual-de-protocolosy.html.

Dra. Sonia Castro; Dr. Gustavo Niotti; Dr. Nestor Robles . (2010). Calidad en la atencion de salud desde los servicios de auditoria . Curso de auditoria medica (págs. 1-10). Uruguay: Hospital Aleman .

Dueñas, A. C. (2008). Medición de la calidad, la eficiencia y la productividad en hospitales publicos detercer nivel de tencion en Bogota. En Revista Universidad \& Empresa, Universidad del Rosario. 17: 203-222.

E. Corral, I. C. (2010). Gestión de calidad del Servicio de Emergencias SAMURProtección Civil. An. Sist. Sanit. Navar. 2010; 33 (Supl. 1): 107-121. 
german chacaltana, m. (2016). calidad en la emergencia. Lima : Reme Arizona .

Grupo de Trabajo SEMES- Insalud. (2001). Calidad en los servicios de urgencias. SEMES, emergencias 2001;13:60-65.

Ing. Luis Pavon; Dra Angelica Garcia . (2017). La auditoria medica como herramienta de control en la Administracion Hospitalaria del Ecuador. Quito- Ecuador : Editor: Dra. Paulina Calvache.

Karlo Celis Solano; Clarita Farias Vilchez . (MARZO de 2018). DETERMINACIÓN E IMPLEMENTACION DE INDICADORES DE CALIDAD PARAMEJORAR LA ATENCION EN LOS SERVICIOS DE EMERGENCIAS. PIURA, LIMA: UNIVERSIDAD DE PIURIA.

Miranda, D. C. (2010). indicadores de calidad en un servicio de emergencias . XXXI congreso nacional de la sociedad española de medicina interna y emergencias . Barcelona: SEMI.

MSP de España. (2010). unidad de urgencias hospitalrias estandares y recomendaciones. españa: Subdirectora General de la Oficina de Planificación Sanitaria y Calidad. AC-SNS. Ministerio de Sanidad y Política Social.

Paz Iglesias-Casarrubios, P. R.-L. (2001). Utilidad de la evaluación de la calidad científico técnica. Rev Calidad Asistencial 2001;16:714-721.

Pérez, M. P., \& Arnesto, M. G. (2015). Calidad asistencial: Concepto, dimensiones y desarrollo operativo. UNEDENSISCIII.

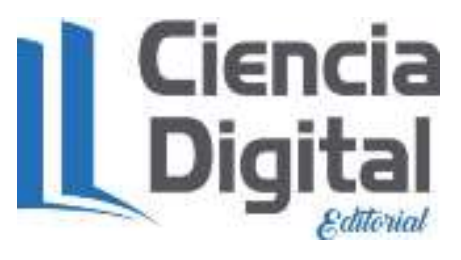




\section{PARA CITAR EL ARTÍCULO INDEXADO.}

Guerrero Lambida, R. (2019). Evaluación de la Calidad Técnica Científico en el Servicio de Emergencia del Hospital Abel Gilbert Pontón Año 2018 - 2019. Ciencia Digital, 3(2.1), 93-107. https://doi.org/10.33262/cienciadigital.v3i2.1.434

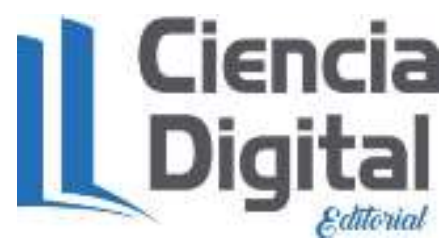

El artículo que se publica es de exclusiva responsabilidad de los autores y no necesariamente reflejan el pensamiento de la Revista Ciencia Digital.

El artículo queda en propiedad de la revista y, por tanto, su publicación parcial y/o total en otro medio tiene que ser autorizado por el director de la Revista Ciencia Digital.
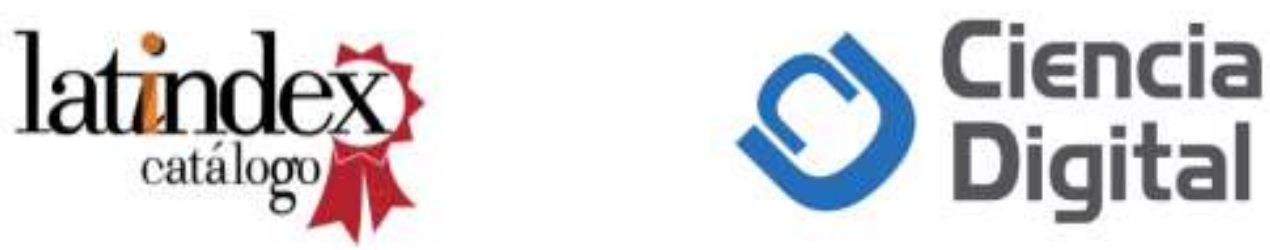